\title{
Oral and Otorhinolaryngological Findings in Adults Who Were Diagnosed With Pediatric Onset Crohn's Disease A Controlled Study
}

\section{Haaramo, Anu}

2019-08

Haaramo , A , Alapulli , H, Aine , L, Tuokkola , J , Saarnisto, U , Roine , R P , Pitkäranta , A \& Kolho , K-L 2019 , ' Oral and Otorhinolaryngological Findings in Adults Who Were Diagnosed With Pediatric Onset Crohn's Disease A Controlled Study ' , Journal of Clinical Gastroenterology , vol. 53 , no. 7 , pp. E269-E275 . https://doi.org/10.1097/MCG.0000000000001074

http://hdl.handle.net/10138/324200

https://doi.org/10.1097/MCG.0000000000001074

cc_by_nc_nd

acceptedVersion

Downloaded from Helda, University of Helsinki institutional repository.

This is an electronic reprint of the original article.

This reprint may differ from the original in pagination and typographic detail.

Please cite the original version. 
Oral and otorhinolaryngological findings in adults who were diagnosed with pediatric onset Crohn's disease: a controlled study

Anu Haaramo ${ }^{\mathrm{b}}$, MD, Heikki Alapulliac, DDS, Liisa Aine ${ }^{\mathrm{d}}$, DDS, PhD, Jetta Tuokkola ${ }^{\mathrm{a}}$, PhD, Ulla Saarnisto $^{\mathrm{ac}}$, DDS, Risto P. Roine ${ }^{\mathrm{ef}}, \mathrm{MD}, \mathrm{PhD}$, Anne Pitkäranta ${ }^{\mathrm{b}}, \mathrm{MD}$, PhD, Kaija-Leena $\mathrm{Kolho}^{\mathrm{a}}$, MD, PhD

Affiliations: ${ }^{a}$ Childrens Hospital, Helsinki University Hospital and Helsinki University, Helsinki, Finland; ${ }^{b}$ Department of Otorhinolaryngology, Head and Neck Surgery, Helsinki University Hospital and Helsinki University, Helsinki, Finland; ${ }^{\mathrm{c} D e p a r t m e n t}$ of Oral and Maxillofacial Diseases, Helsinki University Hospital and Helsinki University, Helsinki, Finland, ${ }^{\mathrm{d} D e p a r t m e n t}$ of Dentistry, Tampere University Hospital and Tampere University, Tampere, Finland, ${ }^{e}$ Department of Health and Social Management, University of Eastern Finland, Kuopio, Finland, ${ }^{\mathrm{f}}$ Group Administration, Helsinki University Hospital and University of Helsinki, Helsinki, Finland.

Address correspondence to: Anu Haaramo, MD, Department of Otorhinolaryngology, Head and Neck Surgery, Helsinki University Hospital, Kasarmikatu 11-13, PO Box 263, 00029 HUS, Helsinki, Finland. Tel: +3589 4711. Fax:+3589 47178286. E-mail: anu.haaramo@hus.fi.

Conflict of Interest and Source of Funding: The authors have no conflicts of interest, relevant to this article to disclose, including financial relationships. This study received external funding from the Foundation for Pediatric Research, the Sigrid Jusélius Foundation and the Foundation for Otorhinolaryngological Research. 


\section{Contributors' Statement:}

Dr Haaramo designed the study, collected the data, carried out the initial analyses, drafted the initial manuscript and revised later versions.

Drs Kolho and Pitkäranta conceptualized and designed the study and critically reviewed and revised the manuscript.

Drs Aine, Alapulli and Saarnisto took part in designing the study, performed the study and reviewed and revised the manuscript.

Dr Tuokkola took part in designing the study, carried out the initial analyses about subjects' nutrition, drafted the initial text on nutrition and reviewed and revised the manuscript.

Dr Roine carried out the initial analyses about the subjects' health-related quality of life and reviewed and revised the manuscript.

All the authors approved the final manuscript as submitted and agree to be accountable for all aspects of the work. 


\begin{abstract}
Background: Up to $50 \%$ of pediatric patients with Crohn's disease (CD) report oral manifestations, but less is known about their oral health when they become adults.

Goals: Our aim was to provide detailed descriptions of the presence of oral and otorhinolaryngological manifestations in pediatric onset CD patients once they reached adulthood, to look for predisposing factors and to compare the findings to matched controls.
\end{abstract}

Study: Adult patients diagnosed with CD in childhood at the Children's Hospital, University of Helsinki, Finland, after 2000 were invited for a follow-up appointment in 2016 and 24 were examined by a dentist and otorhinolaryngologist. They were compared with 22 matched controls from the Population Register Centre. The participants completed questionnaires about their general health, any special diets and their health-related quality of life (HRQoL). Their nutrition was evaluated from food records.

Results: The CD patients had minor oral manifestations at a median of nine years after their childhood diagnosis and the most common was angular cheilitis, which affected six patients and one control, but was not statistically significant $(\mathrm{P}=0.0984)$. $\mathrm{CD}$ with perianal abscessing disease correlated to orofacial findings $(\mathrm{P}=0.0312)$. Most of the patients had normal otorhinolaryngological findings. Subjects with oral lesions had lower mean HRQoL scores than subjects without oral findings and the differences were clinically but not statistically significant. Oral manifestations were not associated with differences in energy intake.

Conclusions: Oral manifestations in adult patients with pediatric onset CD were mild and were not associated with otorhinolaryngological pathology. 
Key words: Crohn's disease; health-related quality of life; inflammatory bowel disease; orofacial granulomatosis; swollen lip 


\section{Introduction}

Crohn's disease (CD) is a chronic inflammatory disease that typically presents in the terminal ileum and colon, but can affect any part of the digestive tract, including the mouth. The reported prevalence of oral manifestations in $\mathrm{CD}$ varies widely and ranges from $0.5 \%$ to $80 \%[1-3]$. These manifestations may coincide with the intestinal symptoms of $\mathrm{CD}$, or precede them, but there are very few systematic studies at different stages of the disease. Furthermore, we are not aware of reports on the otorhinolaryngological health of CD patients.

Orofacial granulomatosis (OFG) is an uncommon chronic inflammation that affects the oral cavity and the area around the mouth without intestinal involvement. Oral lesions in CD and OFG cannot be clinically or histologically distinguished. OFG patients may develop a symptomatic gut disease and clinicians have debated whether OFG is an oral manifestation of CD or a separate inflammatory disorder[4,5]. Our previous follow-up study of 29 patients with pediatric onset OFG found that, $72 \%$ developed symptomatic gut disease within a median of 3.1 years[6]. OFG patients had plenty of oral lesions, but stated that they were mostly asymptomatic. A prospective study of pediatric patients with suspected CD found oral pathology in approximately $40 \%$ of the patients and these mostly went unnoticed before they were examined[7]. This encouraged us to evaluate the presence of oral manifestations in pediatric CD patients when they reached adulthood.

This was a comprehensive cross-sectional study that focused on adult patients with pediatric onset CD who attended Helsinki University Hospital, Finland, for a follow-up visit. Young adults risk dropping out of routine check-ups because of the transition to pediatric to adult care [8] and that is why we chose to study this age group. A dentist and an otorhinolaryngologist examined the patients and the age and sex matched healthy controls and laboratory tests and nutritional evaluation were also carried out. 
The aim of this study was to examine the oral and otorhinolaryngological lesions and symptoms in pediatric onset CD patients when they reached adulthood, evaluate the factors associated with oral manifestations and study the long-term oral health outcomes of CD patients.

\section{Materials and Methods}

This follow-up study was performed between September and December 2016. The study cohort comprised Finnish-speaking adult patients from the Helsinki capital region who were born between 1987-1997 and diagnosed with CD in childhood after the year 2000 at the Helsinki University Children's Hospital, Finland. The diagnosis of CD fulfilled the diagnostic Porto criteria, including endoscopies with biopsies and small bowel imaging[9]. Of the 63 patients we were able to trace, 18 patients could not be contacted and 21 patients declined to participate for unknown reasons, as the ethics committee guidelines state that that we are not allowed to ask people why they don't wish to take part. We also traced 97 healthy controls from the Population Register Centre - approximately four for each patient who agreed to take part - and these were matched for age and sex, but no inclusion or exclusion criteria were applied to the selection. Of these, 47 could not be contacted, 27 declined to participate in the study and one did not respond either way, with these rates being similar to CD patients. The patients and their controls were recruited by sending them a mailed invitation and following this up with a phone call from the study nurse. The final numbers were 24 in the CD group and 22 in the control group.

Study visits

Patients and controls were examined using a structured schema by a dentist (HA, LA or US) and an otorhinolaryngologist (AH). Digital photographs of the mouth were taken to illustrate the findings 
and the orofacial findings were carefully recorded using the oral disease activity score chart for OFG[10]. On the score chart each site in the orofacial area involved was scored individually for activity and type of lesion, with the total score indicating global severity and the maximum total score being 87 . The presence of cobblestoning lesions, fibrous banding/scarring, facial swelling and erythema and staghorning of oral mucosa were recorded. The oral examination was carried out in a dental unit using a dental mirror, dental and periodontal probes, fiber optic illumination and appropriate dental lighting. Radiographs were not taken. The patients' oral hygiene levels were assessed by using the Silness-Löe plaque Index[11]. The periodontal condition was assessed by measuring the depth of the periodontal pockets and the presence of bleeding after probing all the teeth. Dental caries were recorded with the decayed, missing, and filled teeth (DMFT) index[12]. The otorhinolaryngological examination included fiberoscopic laryngoscopy.

\section{Questionnaires}

Subjects filled in questionnaires about their general health, medication, special diets and lifestyle habits. Their health-related quality of life (HRQoL) was evaluated using the 15D questionnaire, a generic, standardized, self-administered measure of HRQoL for individuals aged 16 years or older. The 15D includes the following 15 dimensions: breathing, mental function, speech (communication), vision, mobility, usual activities, vitality, hearing, eating, excretion, sleeping, distress, discomfort and symptoms, sexual activity and depression. There is one question for each of the dimensions and each one has five possible responses. The $15 \mathrm{D}$ can be used as a profile instrument, but it can also provide a single index 15D score, ranging from a maximum of one when there are no problems on any dimension to the minimum of zero when the subject has died[13]. A change of 0.015 in the total $15 \mathrm{D}$ score is considered to be the minimum clinically important difference that a patient can feel[14]. 
Patients were also asked to evaluate the severity of their gut symptoms, and possible orofacial symptoms at the time of the examination, on a Likert-scale with seven response points, where one represented no symptoms and seven represented very difficult symptoms. Patients were asked to keep a food diary for three days before the scheduled appointment and these were checked by a trained nurse for any possible omissions and controversial comments. A dietitian recorded the food diaries by using special food software (AivoDiet, Aivo Finland Oy, Turku, Finland), which makes use of a national database of foods. The food diary also asked about dietary supplements and the compositions of these supplements were checked with the manufacturers. We also recorded whether the patients and the controls consumed any foods containing cinnamon or benzoic acid[15-17], because both of these have been reported to be common allergens when patch testing patients with OFG. Information about the therapies that the patients received were collected from their patient charts.

\section{Laboratory investigations}

Subjects were asked to provide a stool sample so that we could measure their fecal calprotectin in a routine clinical laboratory using a quantitative enzyme immunoassay (PhiCal Test, Calpro AS, Oslo, Norway). Values of $<100 \mu \mathrm{g} / \mathrm{g}$ were considered to be normal[18,19]. Stool samples were collected from 43 subjects: 24 patients and 19 controls.

Blood samples were collected from all subjects and the laboratory tests included C-reactive protein (CRP), the erythrocyte sedimentation rate (ESR), hemoglobin, blood leukocyte counts and antiSaccharomyces cerevisiae antibodies A (ASCAbA) and G (ASCAbG). 
The Ethics Committee of Helsinki University Hospital approved the study protocol and the subjects signed an informed consent form.

Statistical analyses

The non-parametric Mann-Whitney and Spearman's correlation tests and Fisher's exact test were used to investigate the associations between the variables. The groups were compared using the independent samples t-test or Mann-Whitney U test as appropriate. Statistical analyses were performed using GraphPad Prism 7 for Windows (GraphPad Software, Inc, San Diego, USA) and SPSS software version 22 (SPSS Inc., Chicago, Illinois, USA). Differences were considered significant when the P-value was $<0.05$.

\section{Results}

The study group comprised 24 patients with CD and 22 healthy controls matched for age and sex and their background characteristics are shown in Table 1 . The CD patients had a median age of 22.0 years (range 19.3-27.5 years) and the median age of the controls was 21.8 years (range 18.928.9 years). The median duration of the CD patients' follow-up from their primary diagnosis was 9.0 years (range 4.7-15.2 years). Concomitant diseases were recorded in 15 (62.5\%) of the CD patients and four (18.2\%) of the controls (Table 1).

\section{Oral findings}

We found that $19(41.3 \%)$ of the 46 participants had self-reported oral symptoms. Of the $24 \mathrm{CD}$ patients, $14(58.3 \%)$ reported oral symptoms at some point during their follow-up. At the time of 
the study, the patients' subjective evaluation of their own orofacial symptoms varied from a score of one for no symptoms $(n=28)$ to four for moderate symptoms $(n=2)$ and the median score was one for both the patients and the healthy controls $(\mathrm{P}=0.1270$, Table 2$)$. The subjects' own evaluation of their oral symptoms did not correlate with the oral findings observed during the study visit, as the subjective evaluation varied in different people (Spearman's $r=0.1457, \mathrm{n}=46, \mathrm{P}=0.3338$ ).

The patients and controls had very few oral findings (Table 2) and the total scores for OFG, according to the oral disease activity score chart, ranged between zero and three (median 0 ). Of the 46 subjects, $10(21.7 \%)$ had findings in the orofacial area and these comprised eight $(33.3 \%)$ of the 24 patients with CD and two (9.1\%) of the 22 controls. All the oral findings were recorded as mild. The most common oral finding was angular cheilitis in seven $(15.2 \%)$ of the total cohort of 46 subjects, including six with CD, but no statistical significance was reached between the controls and CD patients $(\mathrm{P}=0.0984)$. The oral mucosa, gingival sulcus, gingiva and soft palate were affected in one or two patients each, most with CD, as shown in Table 2. None of the study subjects had cobblestoning, linear ulcers, fibrous banding/scarring, facial swelling and erythema or staghorning of the oral mucosa. In addition, none of the patients had findings in their upper or lower lip, lower gingiva, tongue, floor of the mouth, hard palate or pharynx. Four CD patients who had oral findings at the time of the study had not reported any orofacial symptoms.

We did not observe any significant correlation with age and the total score of the orofacial findings in the whole cohort (Spearman's $\mathrm{r}=-0.2407, \mathrm{n}=46, \mathrm{P}=0.1070$ ) or just among the $\mathrm{CD}$ patients (Spearman's $\mathrm{r}=0.2348, \mathrm{n}=24, \mathrm{P}=0.2695$ ). In addition, the follow-up time from the primary diagnosis of $\mathrm{CD}$ did not correlate with the total score (Spearman's $\mathrm{r}=-0.3059, \mathrm{n}=24, \mathrm{P}=0.1460$ ). However, patients with a history of perianal abscesses had more oral manifestations than patients without perianal disease (Spearman's $\mathrm{r}=0.45, \mathrm{n}=23, \mathrm{P}=0.0312$ ). 
Therapies provided for CD

The therapies that were being provided for $\mathrm{CD}$ at the time of the study are shown in Table 3 . Of the 24 CD patients, $12(50.0 \%)$ were being treated with biological drug therapy and $17(70.8 \%)$ had received biologicals at some point during their follow-up. There were no differences between the oral findings of CD patients who did or did not receive biologicals (Spearman's r=0.01407, n=24, $\mathrm{P}=0.9480$ ). Three patients had not received any medication for $\mathrm{CD}$ at the time of the study and there was no increase in their oral findings compared to the other CD patients ( $\mathrm{P}=0.9481)$.

The CD patients' subjective evaluation of their bowel-related symptoms varied between one for no symptoms ( $n=3)$ to four for moderate symptoms $(n=3)$, with a median score of two. Their selfreported activity for gut symptoms was not associated with more oral findings or a higher total score for orofacial findings (Spearman's $\mathrm{r}=0.1618, \mathrm{n}=24, \mathrm{P}=0.2827$ ).

\section{Dental status}

The oral hygiene level was fair (plaque index $\geq 1.0)$ in $14(30.4 \%)$ of the 46 subjects (median 0.5 , range $0-2.7)$ and untreated dental decay was found in 17 (37.0\%) subjects. Of the 46 subjects, only seven (15.2\%) were cavity free with a DMFT score of zero. All the subjects had gingivitis, except for two, and $10(21.7 \%)$ had periodontal pockets than were at least $4 \mathrm{~mm}$. There were no statistically significant difference in the dental findings between the CD patients and controls. None of the dental findings correlated statistically significantly with the total score of oral manifestations.

\section{Otorhinolaryngological status}

We found that the otorhinolaryngological findings were very minor. Of the 46 subjects, there were $13(28.3 \%)$ who had a noticeable adenoid, including six with CD. In three of the CD subjects this 
was a mucous adenoid. Eight (17.4\%) of the 46 subjects had erythema and four ( $8.7 \%)$ had swelling of the nasal concha. Nasal polyps, atrophy of the nasal conchae or septum perforation were not found. However, $11(23.9 \%)$ of the total cohort reported chronic nasal obstruction and this was as common among the six CD patients as the five controls $(\mathrm{P}=>0.9999)$.

None of the subjects had swelling of the major salivary glands. Six subjects had undergone a tonsillectomy and a tonsillotomy, while the others had grade I-II tonsils. One subject had tonsilloliths as a sign of chronic tonsillitis. Slight enlargement of the cervical lymph nodes was found in three $(6.5 \%)$ of the 46 subjects, but there were no signs of an acute infection in the pharynx. Nasofiberoscopy was successful in all but one subject and there was one case of each of the following: erythema in the vocal cords and erythema and swelling of the arytenoid cartilages. Three subjects had cerumen, which complicated our inspection of their ears, but all the other subjects had normal findings in their ears.

Health-related quality of life

The mean HRQoL scores were lower in patients with CD - with a mean of 0.920 out of a possible score of one - compared with 0.951 for the controls. The difference was regarded as clinically important[14], but not statistically significant. There were slight differences between the groups in the dimensions of vision, breathing, sleeping, speech, excretion, usual activities, distress, depression, discomfort and symptoms and vitality, as shown in Supplementary Figure 1. However, the only statistically significant difference between the CD patients and controls was seen in the excretion dimension $(\mathrm{P}=<0.01)$. When we compared the subjects with oral findings, defined as a total OFG score of $\geq 1$, to those without them, with a total OFG score of zero at the time of the study, this revealed that subjects without oral findings fared better on every HRQoL dimension we measured, except hearing, eating and vitality. However, we only found a statistically significant 
difference in breathing $(\mathrm{P}<0.05)$, with subjects without oral findings having better scores. The disease duration in CD patients did not significantly affect HRQoL.

Laboratory investigations

Twelve (26.1\%) of the subjects had elevated CRP levels over $3 \mathrm{mg} / \mathrm{L}$, but the patients with CD did not have higher CRP levels than the controls, as both of the median levels were $1 \mathrm{mg} / \mathrm{L}(\mathrm{P}=0.1639)$. The CRP values did not correlate with the number of orofacial symptoms, which would have been indicated by higher OFG total scores (Spearmans $r=-0.01183, n=43, P=0.9378$ ). ESR was above the reference values in seven $(15.2 \%)$ of the 46 subjects, with a median of $5 \mathrm{~mm} / \mathrm{h}$ (range 2-66 mm/h), and did not differ markedly between the patients with $\mathrm{CD}$ and the controls, with medians of 8 and $12 \mathrm{~mm} / \mathrm{h}$ respectively $(\mathrm{P}=0.0631)$. Higher ESR correlated with higher total scores for orofacial findings (Spearman's $\mathrm{r}=0.3363, \mathrm{n}=43, \mathrm{P}=0.0239$ ). Blood leukocyte counts did not differ between the $\mathrm{CD}$ patients and controls (median 6.9 and 6.9 respectively, $\mathrm{P}=0.5744$ ) and did not correlate with orofacial symptoms (Spearman's r=0.2133, $\mathrm{n}=43, \mathrm{P}=0.1546$ ).

We found that seven (15.2\%) of the 46 subjects had elevated levels of anti-Saccharomyces cerevisiae antibodies A (ASCAbA) (median titre $30 \mathrm{U} / \mathrm{ml}$, range 23-156) and six of them had CD. Elevated levels of ASCAbG were found in six (13.0\%) subjects (median $30 \mathrm{U} / \mathrm{ml}$, range 27-63), including four who had CD. Elevated ASCAbA or ASCAbG levels did not significantly correlate with the subjects' total score of oral findings (Spearman's $\mathrm{r}=-0.1012, \mathrm{n}=46, \mathrm{P}=0.5033$ and Spearman's r $=0.07197, \mathrm{n}=46, \mathrm{P}=0.6346$ respectively).

Fourteen (32.6\%) of the 43 subjects who provided a stool sample had elevated $(\geq 100 \mu \mathrm{g} / \mathrm{g})$ values of fecal calprotectin (median 308, range 100-2488 $\mu \mathrm{g} / \mathrm{g}$ ). As expected, patients with CD (median 121.5, range 6-2488 $\mu \mathrm{g} / \mathrm{g}$ ) had significantly higher calprotectin values than the controls (median 19, range $3-174 \mu \mathrm{g} / \mathrm{g})(\mathrm{P}=0.002)$, but these were not associated with more oral findings in CD patients 
(Spearman's r=0.1819, $\mathrm{n}=24, \mathrm{P}=0.3948$ ). One healthy control without gut symptoms had fecal calprotectin above the normal range $(174 \mu \mathrm{g} / \mathrm{g})$, but this was back to normal in a couple of weeks and further tests were considered unnecessary.

\section{Nutrition}

Ten CD patients (41.7\%) and seven controls (31.8\%) reported avoiding some food items and avoiding lactose was the most common $(n=7)$. Two other patients $(8.3 \%)$ and one control $(4.5 \%)$ avoided milk and milk products for other health-related reasons and one patient $(4.1 \%)$ and one control (4.5\%) avoided wheat. Four patients (16.7\%) and four controls (18.2\%) reported food allergies. In addition, three patients (12.5\%) and five controls (23.8\%) were either vegetarians or avoided red meat. The differences between CD patients and their controls were not statistically significant. All but three of the 46 subjects had eaten food containing benzoic acid, which is a common allergen that is reported when testing OFG patients, either in its natural forms or as additive. This varied varying from an average of 0.33 to six times per day according their three-day food diaries. We did not find any association between benzoic acid consumption and oral findings $(p=0.734$, one-way ANOVA). In addition, the proportion of subjects who consumed cinnamon did not differ between the CD patients $(37.5 \%)$ and the controls $(31.8 \%, \mathrm{p}=0.686)$. We saw no difference in energy and nutrient intakes between the patients and controls: the median intakes of carbohydrates, fiber and folic acid were lower than recommended in the patients and controls and the median intakes of saturated fatty acids were higher than recommended [20]. CD patients reported higher vitamin D intakes than the controls (median intake 9.7 vs. $5.2 \mu \mathrm{g}$ per day) and this difference was close to statistical significance $(p=0.068$, Mann-Whitney U test, Supplementary Table 1). The oral findings did not correlate with energy intake $\left(\mathrm{r}_{\mathrm{s}}=0.082, \mathrm{p}=0.590\right)$, whereas those with moderate gastrointestinal symptoms received less energy than those with mild or no 
symptoms ( 22.9 vs $33.2 \mathrm{kcal} / \mathrm{kg} /$ day, $\mathrm{p}=0.042$, t-test). However, we saw no statistical difference in their body mass indexes, which were a median of 28.1 in the symptomatic subjects and a median of 23.6 in the subjects with mild or no symptoms $(\mathrm{p}=0.150)$.

Smoking and alcohol consumption

Of the 46 participants, three $(6.5 \%)$ reported smoking cigarettes, one (2.2\%) used an electronic cigarette and seven $(15.2 \%)$ reported taking snuss, which is a moist powder tobacco that is placed under the upper lip for extended periods of time. Taking snuss correlated with more orofacial findings and a higher total score (Spearman's $\mathrm{r}=0.3343, \mathrm{n}=46, \mathrm{P}=0.0232$ ), although the oral findings that were clearly caused by taking snuss, for example damage to the gingival mucosa in the exact place where the subject kept the snuss, were excluded from the total score count. However, smoking did not correlate with more orofacial findings (Spearman's r=-0.1472, $\mathrm{n}=46, \mathrm{p}=0.3288$ ). Alcohol consumption was modest in all of the subjects.

\section{Discussion}

\section{Oral findings}

In previous publications the prevalence of oral manifestations in CD has varied widely and has ranged between $0.5 \%$ and $50 \%$ in adult cohorts[1-3] and $10 \%$ to $80 \%$ in pediatric CD patients [21]. These wide variations can partly be explained by the often subtle character of lesions, the difficulty in noticing lesions if they are not the primary reason for the examination and the fact that the inclusion of non-specific lesions may have varied between studies. More than a third of the 24 pediatric onset CD patients in our study had oral lesions at the time of the study and these were mostly isolated and recorded as mild. The patients were also receiving good therapeutic control for 
their gut disease during the study period, which may have explained the reasonable number of oral lesions. Thus, it seems that pediatric CD only confers a modest risk of oral lesions at adult age, although our study showed that oral lesions were more frequent in pediatric onset CD than in adult onset CD. However, oral lesions were more common in the CD patients than the controls, affecting one-third and one-tenth, respectively.

A study by Laranjeira et al of 113 adult patients with inflammatory bowel disease (IBD) - either ulcerative colitis or CD - and 58 controls, found that oral mucosa lesions increased over the course of the disease[22]. In our cohort, the length of follow up ranged from 4.7 to 15.2 years after the CD diagnosis and the time period was not associated with oral findings. In the Laranjeira et al study, patients in the clinically active phase of IBD were the most affected[22], but this may not have been the case in children[1]. Our study of pediatric onset CD patients who had reached adulthood showed no correlation between the oral findings and the subjective evaluation of the severity of gut symptoms in $\mathrm{CD}$ or with fecal calprotectin levels, a surrogate marker for intestinal inflammation. In our previous study of 29 pediatric onset OFG patients, including $72 \%$ who had $\mathrm{CD}$, there was no association between fecal calprotectin and orofacial findings [6]. This suggests that oral inflammation and intestinal inflammation are driven by different, but uncharacterized, factors.

The oral lesions that are observed in CD patients are typically divided into disease-specific and nonspecific lesions. Specific lesions result from the same disease process as CD in the gut, namely granulomas that can be identified by histology. These specific lesions include swelling of the lips, cheeks and gingiva, cobblestoning of the mucosa, deep linear ulcers and mucosal tags[1,21], which are also typical lesions in OFG. Non-specific lesions are reactive lesions without granulomas[21]. Patients with CD often suffer from aphthous ulceration, angular cheilitis, lip fissuring and 
gingivitis, but these are not specific to $\mathrm{CD}[21]$. In our cohort, disease-specific lesions were not found in any of the subjects and non-specific angular cheilitis was the most frequently seen oral lesion. A 2010 review stated that disease-specific oral manifestations are particularly common in children with $\mathrm{CD}[1]$. Interestingly, it has been suggested that if oral manifestations are present when children are diagnosed with $\mathrm{CD}$ then this might be a marker for a severe $\mathrm{CD}$ phenotype[1]. However, this observation needs to be further examined. A specific risk factor for oral findings in adulthood was a history of perianal abscesses, which is an indicator of a complicated disease subtype[9]. Others have reported such an association between $\mathrm{CD}$ and oral findings[1,2,23].

The spectrum of oral lesions that have been reported in CD patients varies widely. The most common lesion that has been reported is cobblestoning of the buccal mucosa, followed by linear ulcers[24], while the lips[25] and angle of the mouth[26] have been reported to be the most frequently affected sites. In reviews on pediatric patients, mucogingivitis[1] and oral ulceration[21] were reported to be the most common oral lesion. In our cohort, angular cheilitis was the most frequently seen oral lesion, with other lesions being sporadic. In our previous study, the most common site for orofacial lesions in OFG patients was the buccal mucosa, with almost $90 \%$ of patients showing some issues in this area. However, the most common individual oral manifestation was swelling of the lower lip, which was present in about $65 \%$ of our patients[6].

Oral findings are not usually problematic from a clinical point of view, as they may be asymptomatic and they often resolve over time[1]. On the other hand, a number of patients can have very troublesome symptoms that cause pain and functional difficulties in relations to speaking and eating[21] and these require medical intervention or even surgery[2]. In our cohort, oral manifestations at the time of the study were mild, or at most moderate, and did not seem to have a significant effect on the subjects' quality of life. Surprisingly, oral lesions did not affect the eating score of any of the subjects in our study. The HRQoL in CD patients and controls only showed a 
statistically significant difference with regard to excretion functions. CD patients also had lower HRQoL scores in other dimensions, but statistical significance was not reached, which can partially be explained by the relatively small subject groups. Our study was one of the first studies reporting HRQoL in pediatric onset CD patients at adult age.

\section{Treatment}

Earlier publications have showed that oral manifestations are most evident when the gut disease first presents itself and the treatment that the patient receives for this also seems to have an effect on oral lesions in CD patients[21]. In most cases, managing the underlying intestinal disease is sufficient to treat the oral lesions, but variations in the ways that these patients are described and classified makes it hard to compare different patient cohorts. The historical oral lesions that were reported in our study had not received any specific treatment as the patients had just been treated for the gut disease. Half of the patients were on biologicals at the time of our study, and more than twothirds had received them at some point for their gut disease, but this did not seem to affect the orofacial manifestations. We need to examine this finding further to see what it means: does it mean that the biologicals that are administered to treat gut disease are also effective in treating oral lesions or does it mean that that severe gut diseases that require biologicals are not associated with a larger number of oral lesions?

Our previous study of pediatric OFG patients demonstrated the varying severity and need for treatment of oral lesions, as $40 \%$ of the 29 subjects with CD who were receiving systemic medication did not need topical therapy. However, approximately half of the patients who had CD and had received systemic medication for gut symptoms also needed topical treatment for orofacial symptoms[6]. 
To our knowledge, otorhinolaryngological findings in CD patients have not been reported in detail before. Most of our subjects had normal findings during the otorhinolaryngological examination, which included fiberoscopy of the nasopharynx and larynx. Signs of acute or chronic inflammation were not detected, which strengthens the obvious assumption that inflammatory changes in CD are restricted to the gastrointestinal tract without excess otorhinolaryngological comorbidity.

Empirically, CD patients seem to have suffered quite commonly from nasal obstruction, but in our study neither the patients' own evaluation of any nasal obstruction, nor the clinical findings of the nasal cavity and nasal conchae, differed significantly between the CD patients and controls. In addition, no nasal polyps that caused nasal obstruction were detected. A detectable adenoid was as common in the CD patients as in the controls, but interestingly all patients with a mucous adenoid had CD.

\section{Blood markers}

In our earlier study of 29 patients with pediatric onset OFG, ASCAbA appeared to be a possible marker for the development of CD in OFG patients[6]. In this current study, ASCAbA did not correlate with oral findings in pediatric onset adult CD patients in a statistically significant manner, but overall there were fewer oral findings in this study cohort.

Only a small percentage of patients had elevated CRP levels and these did not correlate with orofacial findings, unlike elevated ESR, which was found in one-sixth of the patients. However, in our previous study of 29 patients with pediatric onset OFG[6], elevated CRP or ESR levels were not connected to oral disease activity[27]. Elevated ESR and CRP have previously been connected to a higher risk of CD in OFG patients[15], but OFG has not been associated with any significant hematologic abnormality [4,28]. 


\section{Nutrition}

Patients with CD have poor nutritional absorption and their dietary intake of several nutrients may be lower than recommended, due to them self-selecting an elimination diet in an attempt to relieve their symptoms. This means that patients are at nutritional risk[29]. In our study, the median intakes of carbohydrates, fiber and folic acid were lower in both the patients and controls and the median intakes of saturated fatty acids were higher than recommended [20] (Supplementary Table 3). There has been much debate about the role of vitamin D in immunology in the press and social media, which was probably reflected in the fact that the patients received more vitamin $\mathrm{D}$ than the controls. The mild oral findings, or perceived oral symptoms, were not associated with energy intake, whereas the gut symptoms seemed to be associated with eating less. However, this was not reflected in the body mass index of the subjects, suggesting underreporting in the food records, which is a common finding when studying nutrition[30].

Strengths and limitations

We believe that the number of patients that were included was a limitation in our study, even though they were collected from a large population of 1.1 million inhabitants. It seems that young adults have college or work obligations that get in the way of them taking part in studies and this is a challenge when it comes to recruiting study subjects. A valid strength of our study was that it was comprehensive and included a dental and otorhinolaryngological examination, laboratory tests, nutritional aspects and HRQoL measurements. Including a control group in the study was also a valid strength of this study. To minimize inter-observer variations, patients were examined in parallel by a designated otorhinolaryngologist and a dentist using a structured schema. However, the dental examination was provided at no cost, which might have encouraged people with poorer 
dental conditions to participate, and this might have explained the high number of patients with gingivitis.

\section{Conclusion}

There are many kinds of orofacial manifestations in CD patients and they also vary between individuals. Oral manifestations do not seem to increase during the course of the gut disease and they seem to be inactive when the gut disease is under good therapeutic control. The possible exacerbation of oral lesions during the active phases of the gut disease need to be further examined. Dental findings did not correlate with oral lesions in our study and otorhinolaryngological comorbidity was not found. Oral manifestations were more likely to be seen in CD patients with a history of perianal abscesses, but other distinct factors associated with oral manifestations were not observed.

\section{Acknowledgements}

We are grateful to research nurse Anne Nikkonen for organizing the study and recruiting the patients, Rhea Paajanen for collecting the blood samples and Heidi Hirsimäki for recording the food diaries. 


\section{References}

1. Rowland M, Fleming P, Bourke B. Looking in the mouth for Crohn's disease. Inflamm Bowel Dis. 2010;16:332-337.

2. Katsanos KH, Torres J, Roda G, et al. Review article: non-malignant oral manifestations in inflammatory bowel diseases. Aliment Pharmacol Ther. 2015;42:40-60.

3. Tan CX, Brand HS, de Boer NK, et al. Gastrointestinal diseases and their oro-dental manifestations: Part 1: Crohn's disease. Br Dent J. 2016;221:794-799.

4. Sanderson J, Nunes C, Escudier M, et al. Oro-facial granulomatosis: Crohn's disease or a new inflammatory bowel disease? Inflamm Bowel Dis. 2005;11:840-846.

5. Challacombe SJ. Oro-facial granulomatosis and oral Crohns disease: are they specific diseases and do they predict systemic Crohns disease? Oral Dis. 1997;3:127-129.

6. Haaramo A, Alapulli H, Aine L, et al. Detailed Follow-up Study of Pediatric Orofacial Granulomatosis Patients. J Pediatr Gastroenterol Nutr. 2017;65:388-393.

7. Harty S, Fleming P, Rowland M, et al. A prospective study of the oral manifestations of Crohn's disease. Clin Gastroenterol Hepatol. 2005;3:886-891.

8. Cole R, Ashok D, Razack A, et al. Evaluation of Outcomes in Adolescent Inflammatory Bowel Disease Patients Following Transfer From Pediatric to Adult Health Care Services: Case for Transition. J Adolesc Health. 2015;57:212-217.

9. Levine A, Koletzko S, Turner D, et al. ESPGHAN revised porto criteria for the diagnosis of inflammatory bowel disease in children and adolescents. J Pediatr Gastroenterol Nutr.

2014;58:795-806.

10. White A, Nunes C, Escudier M, et al. Improvement in orofacial granulomatosis on a cinnamonand benzoate-free diet. Inflamm Bowel Dis. 2006;12:508-514.

11. SILNESS J, LOE H. Periodontal Disease in Pregnancy. Ii. Correlation between Oral Hygiene and Periodontal Condtion. Acta Odontol Scand. 1964;22:121-135.

12. . World Health Organization. Oral Health surveys. Basic methods. 1997.

13. Sintonen H. The 15D instrument of health-related quality of life: properties and applications. Ann Med. 2001;33:328-336.

14. Alanne S, Roine RP, Rasanen P, et al. Estimating the minimum important change in the 15D scores. Qual Life Res. 2015;24:599-606.

15. Campbell H, Escudier M, Patel P, et al. Distinguishing orofacial granulomatosis from crohn's disease: two separate disease entities? Inflamm Bowel Dis. 2011;17:2109-2115. 
16. Fitzpatrick L, Healy CM, McCartan BE, et al. Patch testing for food-associated allergies in orofacial granulomatosis. J Oral Pathol Med. 2011;40:10-13.

17. Wray D, Rees SR, Gibson J, et al. The role of allergy in oral mucosal diseases. QJM. 2000;93:507-511.

18. Kolho KL, Raivio T, Lindahl H, et al. Fecal calprotectin remains high during glucocorticoid therapy in children with inflammatory bowel disease. Scand J Gastroenterol. 2006;41:720-725.

19. Hamalainen A, Sipponen T, Kolho KL. Infliximab in pediatric inflammatory bowel disease rapidly decreases fecal calprotectin levels. World J Gastroenterol. 2011;17:5166-5171.

20. Nordic council of ministers. Nordic Nutrition Recommendations 2012, 2012. Available at: http://dx.doi.org/10.6027/Nord2014-002.

21. Skrzat A, Olczak-Kowalczyk D, Turska-Szybka A. Crohn's disease should be considered in children with inflammatory oral lesions. Acta Paediatr. 2017;106:199-203.

22. Laranjeira N, Fonseca J, Meira T, et al. Oral mucosa lesions and oral symptoms in inflammatory bowel disease patients. Arq Gastroenterol. 2015;52:105-110.

23. Hussey S, Fleming P, Rowland M, et al. Disease outcome for children who present with oral manifestations of Crohn's disease. Eur Arch Paediatr Dent. 2011;12:167-169.

24. Basu MK, Asquith P, Thompson RA, et al. Proceedings: Oral lesions in patients with Crohn's disease. Gut. 1974; 15:346.

25. Plauth M, Jenss H, Meyle J. Oral manifestations of Crohn's disease. An analysis of 79 cases. $J$ Clin Gastroenterol. 1991;13:29-37.

26. Lisciandrano D, Ranzi T, Carrassi A, et al. Prevalence of oral lesions in inflammatory bowel disease. Am J Gastroenterol. 1996;91:7-10.

27. Haaramo A, Alapulli H, Aine L, et al. Detailed Follow-up Study of Pediatric Orofacial Granulomatosis Patients. J Pediatr Gastroenterol Nutr. 2017;65:388-393.

28. Al Johani KA, Moles DR, Hodgson TA, et al. Orofacial granulomatosis: clinical features and long-term outcome of therapy. J Am Acad Dermatol. 2010;62:611-620.

29. Hartman C, Marderfeld L, Davidson K, et al. Food Intake Adequacy in Children and Adolescents With Inflammatory Bowel Disease. J Pediatr Gastroenterol Nutr. 2016;63:437-444.

30. Buzzard M. 24-hour dietary recall and food record methods. In: Willet W, ed., Nutritional Epidemiology. New York, USA: Oxford university press; 1998:50-73. 
Table legends:

Table 1. Background characteristics of the study population.

Table 2. Site and type of oral manifestations in patients with CD and controls and the participants' own evaluation of their orofacial symptoms at the time of the study.

Table 3. Therapy given to patients with CD at the time of the study.

\section{Supplemental Digital Contents:}

Supplementary Figure 1. Health-related quality of life in CD patients and controls, as evaluated by the $15 \mathrm{D}$ questionnaire.

Supplementary Table 1. Median (interquartile range) intakes of energy and nutrients in CD patients and controls. 
Table 1. Background characteristics of the study population

\begin{tabular}{|c|c|}
\hline Number of subjects & 46 \\
\hline Patients with CD & $24(52.2 \%)$ \\
\hline Male & $14(58.3 \%)$ \\
\hline Healthy controls & $22(47.8 \%)$ \\
\hline Male & $11(50.0 \%)$ \\
\hline \multicolumn{2}{|l|}{ Age in years, median (range) } \\
\hline At Crohn's disease diagnosis & $13(8-16)$ \\
\hline \multicolumn{2}{|l|}{ At study appointment } \\
\hline Patients with CD & $22(19-28)$ \\
\hline Healthy controls & $22(19-29)$ \\
\hline \multicolumn{2}{|l|}{ Diagnoses } \\
\hline $\mathrm{CD}$ & 24 \\
\hline Previous surgery & 5 \\
\hline Fistulazing perianal disease & 7 \\
\hline \multicolumn{2}{|l|}{ Concomitant diseases, CD patients } \\
\hline Pollen and animal allergies or atopy & 8 \\
\hline Depression & 3 \\
\hline Psoriasis & 2 \\
\hline Asthma & 1 \\
\hline Migraine & 1 \\
\hline Complement $\mathrm{C} 4 \mathrm{~b}$ deficiency & 1 \\
\hline Ikhthyosis & 1 \\
\hline Disorders of vitamin D metabolism & 1 \\
\hline Deficit in attention, motor control and perception & 1 \\
\hline \multicolumn{2}{|l|}{ Concomitant diseases, controls } \\
\hline Rheumatoid arthritis & 2 \\
\hline Asthma & 1 \\
\hline Hypothyreosis & 1 \\
\hline Rhinitis chronica & 1 \\
\hline Acne & 1 \\
\hline \multicolumn{2}{|l|}{ Duration of $C D$ in years, median (range) } \\
\hline Time between CD diagnosis and study & $9.0(4.7-15.0)$ \\
\hline $\mathrm{CD}=$ Crohn's disease & \\
\hline
\end{tabular}


Table 2. Site and type of oral manifestations in patients with CD and controls and the participants' own evaluation of their orofacial symptoms at the time of the study. 


\begin{tabular}{|c|c|c|c|}
\hline & All & $C D$ & Controls \\
\hline Number of study subjects & 46 & 24 & 22 \\
\hline Lips & 0 & 0 & 0 \\
\hline Lower lip & 0 & 0 & 0 \\
\hline Upper lip & 0 & 0 & 0 \\
\hline Angular cheilitis* & 7 & 6 & 1 \\
\hline Oral mисова & 1 & 1 & 0 \\
\hline Ulceration & 1 & 1 & 0 \\
\hline Fissures & 1 & 1 & 0 \\
\hline Buccal sulcus & 2 & 2 & 0 \\
\hline Erythema & 1 & 1 & 0 \\
\hline Ulceration & 1 & 1 & 0 \\
\hline Gingiva & 2 & 1 & 1 \\
\hline Erythema & 2 & 1 & 1 \\
\hline Tongue & 0 & 0 & 0 \\
\hline Floor of the mouth & 0 & 0 & 0 \\
\hline Soft palate & 1 & 1 & 0 \\
\hline Nodules & 1 & 1 & 0 \\
\hline Hard palate & 0 & 0 & 0 \\
\hline Pharynx & 0 & 0 & 0 \\
\hline Total score $^{* *}$ median (range) & $0(0-3)$ & $0(0-3)$ & $0(0-1)$ \\
\hline $\begin{array}{l}\text { Participants own evaluation of oral symptoms } * * * \\
\text { Median (range) }\end{array}$ & $1(1-4)$ & $1(1-4)$ & $1(1-4)$ \\
\hline $\begin{array}{l}\mathrm{CD}=\text { Crohn's disease } \\
* \mathrm{P}=0.0984 \text { when compared between patients with } \mathrm{C} \\
* * \text { Oral disease activity score chart for orofacial gran } \\
* * * \text { Likert scale from } 1 \text { (no symptoms) to } 7 \text { (difficult }\end{array}$ & $\begin{array}{l}\text { controls } \\
\text { osis } \\
\text { oms) }\end{array}$ & & \\
\hline
\end{tabular}


Table 3. Therapy given to patients with CD at the time of the study.

\begin{tabular}{|lc|}
\hline Number of subjects & 24 \\
\hline Systemic treatment & $12(50.0 \%)$ \\
Biological drug therapy & $11(45.8 \%)$ \\
$\quad$ anti-TNF alfa therapy & $1(4.2 \%)$ \\
$\quad$ anti-IL12/23 antibody & $9(37.5 \%)$ \\
Azathioprine/6-merkaptopurine & $4(16.6 \%)$ \\
$\quad$ as monotherapy & $5(20.8 \%)$ \\
$\quad$ combined with a biological drug & $5(20.8 \%)$ \\
M-aminosalicylic acid & $1(4.2 \%)$ \\
No medication for CD & $3(12.5 \%)$ \\
\hline CD= Crohn's disease & \\
\hline
\end{tabular}


Supplementary Figure 1. Health-related quality of life in CD patients and controls, as evaluated by the $15 \mathrm{D}$ questionniare.

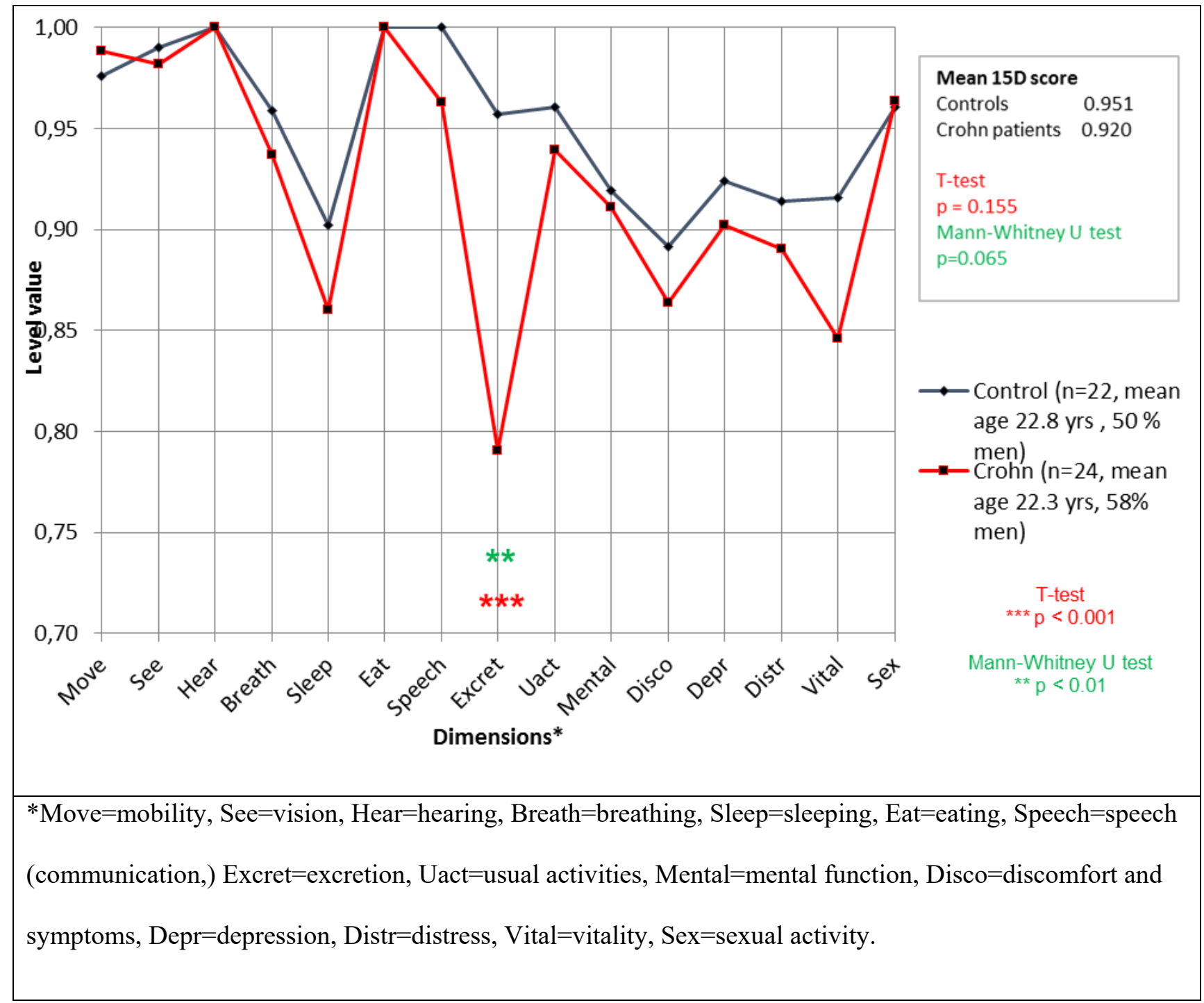


Supplementary Table 1. Median (interquartile range) intakes of energy and nutrients in CD patients and controls. 


\begin{tabular}{|c|c|c|c|c|c|}
\hline & $C D$ patients & Controls & $\begin{array}{c}P \text {-value } \\
\text { for } \\
\text { difference* }\end{array}$ & $\begin{array}{c}R N I^{* *} \\
\text { Female }\end{array}$ & Males \\
\hline Energy (kcal) & $2334.0(1849.2-2658.9)$ & $2328.5(1958.4-2575.9)$ & 0.895 & & \\
\hline Protein (g) & $100.1(80.1-140.0)$ & $78.3(68.8-109.4)$ & 0.104 & & \\
\hline Protein $(\mathrm{E} \%)$ & $17.5(15.5-22.1)$ & $14.3(12.4-18.4)$ & 0.079 & \multicolumn{2}{|c|}{$10-20$} \\
\hline Carbohydrates (g) & $247.3(184.9-315.4)$ & $254.2(208.4-303.0)$ & 0.860 & & \\
\hline Carbohydrates (E\%) & $40.9(36.0-46.4)$ & $43.5(40.0-50.4)$ & 0.235 & \multicolumn{2}{|c|}{$45-60$} \\
\hline Fat $(g)$ & $97.9(71.4-106.5)$ & $87.9(70.6-113.1)$ & 0.758 & & \\
\hline Fat $(\mathrm{E} \%)$ & $36.6(31.2-41.4)$ & $35.5(30.8-41.3)$ & 0.598 & \multicolumn{2}{|c|}{$25-40$} \\
\hline SAFA (g) & $28.6(20.7-37.1)$ & $30.9(20.9-41.1)$ & 0.598 & & \\
\hline SAFA (E\%) & $11.7(9.3-14.0)$ & $11.2(8.9-15.0)$ & 0.947 & \multicolumn{2}{|c|}{$>10$} \\
\hline MUFA (g) & $33.6(25.7-37.1)$ & $29.6(21.2-43.4)$ & 0.676 & & \\
\hline MUFA (E\%) & $12.9(10.5-14.1)$ & $12.0(9.0-16.2)$ & 0.509 & \multicolumn{2}{|c|}{$10-20$} \\
\hline PUFA (g) & $13.7(11.9-8.0)$ & $14.2(9.4-19.4)$ & 0.783 & & \\
\hline PUFA (E\%) & $5.9(4.6-6.8)$ & $6.0(4.1-7.6)$ & 0.775 & \multicolumn{2}{|c|}{$5-10$} \\
\hline Sucrose (g) & $50.6(18.8-62.5)$ & $42.3(31.4-54.4)$ & 0.509 & & \\
\hline Sucrose (E\%) & $8.4(4.4-11.8)$ & $7.4(6.0-8.8)$ & 0.792 & \multicolumn{2}{|l|}{$>10$} \\
\hline Fiber $(\mathrm{g})$ & $17.4(13.5-22.4)$ & $20.1(15.9-25.9)$ & 0.191 & \multicolumn{2}{|c|}{$25-35$} \\
\hline Vitamin A $(\mu \mathrm{g})$ & $703.0(461.4-854.1)$ & $747.8(452.3-907.6)$ & 0.826 & 900 & 700 \\
\hline Vitamin D $(\mu \mathrm{g})$ & $9.7(6.1-15.1)$ & $5.2(3.7-12.2)$ & 0.068 & \multicolumn{2}{|c|}{10} \\
\hline Vitamin E (mg) & $12.0(10.1-16.4)$ & $12.0(9.4-14.7)$ & 0.416 & 10 & 8 \\
\hline Vitamin $\mathrm{K}(\mu \mathrm{g})$ & $121.4(86.0-199.9)$ & $101.9(70.8-132.7)$ & 0.129 & & \\
\hline Vitamin C (mg) & $112.9(56.9-209.4)$ & $95.9(59.3-152.3)$ & 0.598 & \multicolumn{2}{|c|}{75} \\
\hline Thiamine (mg) & $1.3(0.9-2.0)$ & $1.1(1.0-1.6)$ & 0.350 & 1.4 & 1.1 \\
\hline Riboflavin (mg) & $2.2(1.4-3.9)$ & $1.8(1.4-2.2)$ & 0.180 & 1.6 & 1.3 \\
\hline Niacine (mg) & $50.6(31.0-65.6)$ & $39.3(29.3-55.2)$ & 0.180 & 19 & 15 \\
\hline Pyridoxine (mg) & $2.9(1.7-4.6)$ & $2.1(1.8-3.9)$ & 0.403 & 1.6 & 1.3 \\
\hline Vitamin B12 $(\mu \mathrm{g})$ & $6.8(4.6-10.8)$ & $5.0(3.3-7.3)$ & 0.104 & \multicolumn{2}{|c|}{2.0} \\
\hline Folic acid $(\mu \mathrm{g})$ & $271.1(214.8-365.3)$ & $277.0(228.2-404.0)$ & 0.692 & 300 & 400 \\
\hline Magnesium (mg) & $349.9(260.6-494.0)$ & 363.8 (308.7-447.7) & 0.843 & 350 & 280 \\
\hline Calcium (mg) & $1011.2(727.5-1306.8)$ & $957.3(772.7-1217.1)$ & 0.644 & \multicolumn{2}{|c|}{800} \\
\hline Iron (mg) & $12.0(9.9-15.6)$ & $13.7(11.8-15.8)$ & 0.244 & 9 & 15 \\
\hline Potassium (mg) & $3499.0(2838.5-4428.8)$ & $3533.1(3028.6-4147.6)$ & 0.692 & 3500 & 3100 \\
\hline Phosphorus (mg) & $1441.7(1172.9-2064.6)$ & $1457.7(1186.1-1805.0)$ & 0.792 & \multicolumn{2}{|c|}{600} \\
\hline Selenium $(\mu \mathrm{g})$ & $83.3(66.4-115.2)$ & $60.4(52.1-105.8)$ & 0.079 & 60 & 50 \\
\hline Zinc (mg) & $12.3(9.7-17.8)$ & $12.1(10.3-16.8)$ & 0.930 & 9 & 7 \\
\hline
\end{tabular}


